\title{
Role of engulfment and cell motility 1 (ELMO1) gene polymorphism in development of diabetic kidney disease
}

\author{
Thoria A. Omar ${ }^{1 *}$, Shimaa K. Zewain², Mohamed M. Ghonaim³ ${ }^{3}$ Khadija A. Refaat ${ }^{1}$ and Dalia H. Abou-Elela ${ }^{1}$
}

\begin{abstract}
Background: Diabetic kidney disease (DKD) is a progressive kidney disease that affects diabetic patients irrespective of glycemic state or hypertension. Therefore, early detection of DKD is of critical importance. Many genome-wide association studies have identified the engulfment and cell motility 1 (ELMO1) gene as a genetic marker linked to DKD. This study aimed to investigate the association between ELMO1 rs741301 gene polymorphism and the development of DKD among Egyptian patients with type 2 diabetes mellitus (T2DM). Allele and genotype frequencies were investigated in 304 subjects by real-time PCR allelic discrimination assay: 100 DKD patients, 102 diabetic patients without DKD, and 102 healthy controls.

Results: GG genotype of ELMO1 (rs741301) SNP and its allele frequencies were significantly high in all diabetic patients. GG genotype had an odds ratio (OR) of 6.095 and 95\% confidence interval (Cl) of 2.456-15.125, $p<0.001$, while the frequent allele $\mathrm{G}$ had an $\mathrm{OR}$ of 2.366 and $95 \% \mathrm{Cl}$ of $1.450-3.859, p=0.001$. No significant difference was observed between T2DM without DKD and DKD.
\end{abstract}

Conclusion: Our results could not establish an association between the ELMO1 rs741301 variant and the progression of DKD.

Keywords: Type 2 diabetes mellitus, Diabetic kidney disease, Single-nucleotide polymorphism, ELMO1, Real-time $P C R$

\section{Background}

Type 2 diabetes mellitus (T2DM) is a public health problem threatening the economies of all nations, especially developing countries. The International Diabetes Federation (IDF) listed Egypt among the world's top 10 countries with the highest number of patients with diabetes. The prevalence of diabetes in adults is $15.2 \%$, with the total number of diabetes cases in adults amounting to $8,850,400$ [1].

Diabetic kidney disease (DKD) is one of the most common microvascular complications of diabetic mellitus $(\mathrm{DM})$ and is the primary cause of end-stage renal disease

\footnotetext{
* Correspondence: THURAYA.OMAR@med.menofia.edu.eg

${ }^{1}$ Clinical Pathology Department, Faculty of Medicine, Menoufia University, Shebin El-Kom, Egypt

Full list of author information is available at the end of the article
}

(ESRD), which results in high morbidity and mortality [2]. DKD is manifested by a progressive deterioration in the glomerular filtration rate, increased urinary albumin excretion, increased thickness of the basement membrane, and mesangial expansion with the accumulation of extracellular matrix (ECM) proteins [3].

Several pathological processes are involved in the pathogenesis of DKD in patients with T2DM. The increases in the mitochondrial generation of reactive oxygen species (ROS) and in the cellular expression of transforming growth factor beta (TGF- $\beta$ ) generate apoptosis within renal glomerular cells, which are important effects of hyperglycemia [4]. ROS activate signaling molecules and transcription factors, leading to enhanced expression of cytokines, growth factors, and ECM proteins, macrophage infiltration, and overproduction of leucocyte

\section{Springer Open}

(c) The Author(s). 2021 Open Access This article is licensed under a Creative Commons Attribution 4.0 International License, which permits use, sharing, adaptation, distribution and reproduction in any medium or format, as long as you give appropriate credit to the original author(s) and the source, provide a link to the Creative Commons licence, and indicate if changes were made. The images or other third party material in this article are included in the article's Creative Commons licence, unless indicated otherwise in a credit line to the material. If material is not included in the article's Creative Commons licence and your intended use is not permitted by statutory regulation or exceeds the permitted use, you will need to obtain permission directly from the copyright holder. To view a copy of this licence, visit http://creativecommons.org/licenses/by/4.0/. 
adhesion molecules in the kidneys, which contribute to cellular hypertrophy and increased collagen synthesis [5].

Therefore, it is urgent to create new, efficient, and safe therapeutic approaches against DKD based on understanding the molecular mechanisms of the disease [6].

Various genetic variants and environmental factors are involved in the etiology of DKD. However, the exact mechanism regarding the pathophysiology of DKD remains unclear [7]. Clinical and epidemiological studies have shown that there is a family history of DKD in various ethnic groups, suggesting that genetic factors play a key role in developing the disease $[8,9]$.

The engulfment and cell motility 1 (ELMO1) gene is a protein made up of 720 amino acids, located on chromosome 7p14.2-14.1, and encodes a member of the engulfment and cell motility protein family. ELMO1 interacts with the dedicator of cytokinesis proteins (Dock180) and functions as a guanine nucleotide exchange factor for the small GTPase Rac1, thus regulating cell migration and promoting phagocytosis [10]. ELMO1 has been proven to protect endothelial cells from apoptosis by stimulating the Rac1/PAK/AKT signaling cascade in vitro and in vivo [11].

Genome-wide association studies (GWAS) suggest that polymorphism within human ELMO1 has a significant pathophysiological role in the development of albuminuria and the fibrotic tissue changes characteristic of DKD. Mutation in the ELMO1 gene is associated with increases in renal expression of genes encoding transforming growth factor beta 1 (TGF- $\beta 1$ ), endothelin 1 , and $\mathrm{NAD}(\mathrm{P}) \mathrm{H}$ oxidase, which is implicated in fibrogenesis and epithelial-mesenchymal transition [12].

Several genetics studies were conducted on different ethnic populations to demonstrate the association between ELMO1 gene variant and DKD. Japanese [13], American, Indian [14], European American [15], and Chinese [16] studies found that variants in the ELMO1 gene are related to kidney diseases in T2DM, whereas other studies failed to find any correlation between ELMO1 and development of DKD among other ethnic populations [17, 18].

In Egypt, the incidence of T2DM and its renal complications have increased, with no conclusive data on the role of ELMO1 in DKD pathogenesis among T2DM patients. Thus, this study aims to demonstrate the association between genetic polymorphism of the rs741301 ELMO1 gene variant and the development of DKD in Egyptian patients with T2DM.

\section{Methods}

A case-control study was carried outin clinical pathology departement faculty of medicine, menoufia university during the period from March 2019 to May 2020. The patients were selected from the Outpatient Endocrinology Clinic, Internal Medicine Department.

The patients were classified according to the diagnostic criteria of the American Diabetes Association (ADA) for DM and DKD [19] as follows: Group 1 included 100 DKD patients with early morning spot albumin to creatinine ratio (ACR) of $>30 \mathrm{mg} / \mathrm{g}$ creatinine, Group 2 included 102 diabetic patients without DKD and ACR of $<30 \mathrm{mg} / \mathrm{g}$ creatinine, and Group 3 included 102 healthy individuals as age- and gender-matched controls to detect wild genotypes in Egyptian population. All volunteers had no kidney disease or any acute or chronic illness. Exclusion criteria were as follows: patients with type $1 \mathrm{DM}$, recent urinary tract infection, albuminuria with unknown cause, uncontrolled hypertension, congestive heart failure, and other endocrine disease and pregnant patients. All groups were subjected to the following examinations and testing.

\section{Complete clinical examination}

Weight, height, waist circumference, hip circumferenceand blood pressure were measured, body mass index (BMI) was calculated as the weight in kilograms divided by the square of the height in meters $\left(\mathrm{kg} / \mathrm{m}^{2}\right)$, and waistto-hip ratio (WHR) was calculated.

\section{Sampling}

Four milliliters of the venous blood was collected after 12-hour fasting and divided as follows: in Tube A, $2 \mathrm{ml}$ of blood was left to clot and serum was separated by centrifugation at $1000 \times g$ for $10 \mathrm{~min}$ for immediate assay of lipid profile, fasting blood sugar (FBS), and serum creatinine; in Tube B, $2 \mathrm{ml}$ of whole blood was collected on ethylenediaminetetraacetic acid (EDTA) tube and then divided into two aliquots, both kept at $-20^{\circ} \mathrm{C}$, one used for the determination of $\boldsymbol{E L M O 1}$ (rs741301) genotypes and the other for the determination of glycated hemoglobin (HbA1c). After 2 hour of eating, a blood sample was obtained to measure 2-hour postprandial blood glucose (2-hPPG).

\section{Urine specimen collection}

Random urine samples were collected in sterile containers to estimate urine creatinine and albumin and calculate ACR [20].

\section{Laboratory tests}

FBS, 2-hPPG, serum, urine creatinine, and lipid profile [total cholesterol (TC), triglycerides (TG), lowdensity lipoprotein cholesterol (LDL-C), and highdensity lipoprotein cholesterol (HDL-C)] were measured by autoanalyzer AU680 (Beckman Coulter, USA). LDL-C concentration was calculated according 
to the Friedewald formula. Quantitative colorimetric measurement of glycated hemoglobin (HbA1c) was calculated as a percentage of total hemoglobin using kits supplied by BIOTEC (London W1G9QR, UK). Morning clear midstream urine samples (10-20 ml) were collected to obtain the albumin/creatinine ratio. Samples were centrifuged at $3000 \mathrm{rpm}$ for $10 \mathrm{~min}$. Creatinine was measured by AU680. Albumin levels were measured using an immunoturbidimetric method by the HEALES microalbumin test kit (Shenzhen Huisong Technology Development, China). Urinary ACR (milligram/gram (mg/g)) was calculated. eGFR was estimated according to the Chronic Kidney Disease Epidemiology Collaboration (CKD-EPI) equation [21]. The atherogenic index of plasma (AIP) was calculated as a logarithmic transformation of the ratio of TG to HDL-C [22].

Determination of ELMO1 (rs741301) genotypes by realtime PCR

\section{Genomic DNA extraction [23]}

Using a Thermo Scientific DNA isolation kit (Thermo Scientific GeneJET Whole Blood Genomic DNA Purification), we extracted genomic DNA. The DNA quantities were examined with a spectrophotometer (Implen NanoPhotometer ${ }^{\mathrm{Tm}}$ N60 UV/VIS spectrophotometer, Germany). The extracted DNA was stored in aliquots at $-80^{\circ} \mathrm{C}$ until performing the PCR.

\section{Taqman SNP genotyping assay [24]}

The ELMO1 single-nucleotide polymorphism (SNP) (rs741301) was genotyped using a real-time PCR system (Rotor-Gene, Applied Biosystems, Foster City, USA): the sense primer, 5'-GCAGTTCCCATGGTGG TTATCATTA-3'; the antisense primer, 5'-TGAACT CTTCAAGCTCAATAGCAATAGATT-3', using fluorescent-labeled probes [VIC/VAM] AGCAATAG ATTTTATGAGGTGGTGG[A/G] TTCCAGAGGT TATGTTATCACTAAT and TaqMan Master Mix (Applied Biosystems, Foster City, CA). The total volume reaction of $20 \mu \mathrm{l}$ was reached by mixing $1.25 \mu \mathrm{l}$ of the probe, $10 \mu \mathrm{l}$ of Master Mix, and $0.75 \mu \mathrm{l}$ of DNAase-free water. For every sample, $8 \mu$ l of DNA template and $8 \mu \mathrm{l}$ of nuclease-free water for the negative control were added. The PCR conditions were as follows: initial denaturation was done at $95^{\circ} \mathrm{C}$ for 15 min, followed by 50 cycles of denaturation at $94^{\circ} \mathrm{C}$ for $1 \mathrm{~min}$, primer annealing at $60^{\circ} \mathrm{C}$ for $1 \mathrm{~min}$, then primer extension at $74^{\circ} \mathrm{C}$ for $2 \mathrm{~min}$, and the final extension step at $72^{\circ} \mathrm{C}$ for $1 \mathrm{~min}$. The allele distribution and amplification plots of rs741301 were demonstrated in Fig. 1a and b.

\section{Statistical analysis}

All statistical calculations were conducted using SPSS version 23 (SPSS Inc., Released 2015. IBM SPSS Statistics for Windows, Version 23.0, Armonk, NY: IBM Corp.). Data were expressed as mean $(\mathbf{x})$, standard deviation (SD), number (No.), and percentage (\%). ANOVA

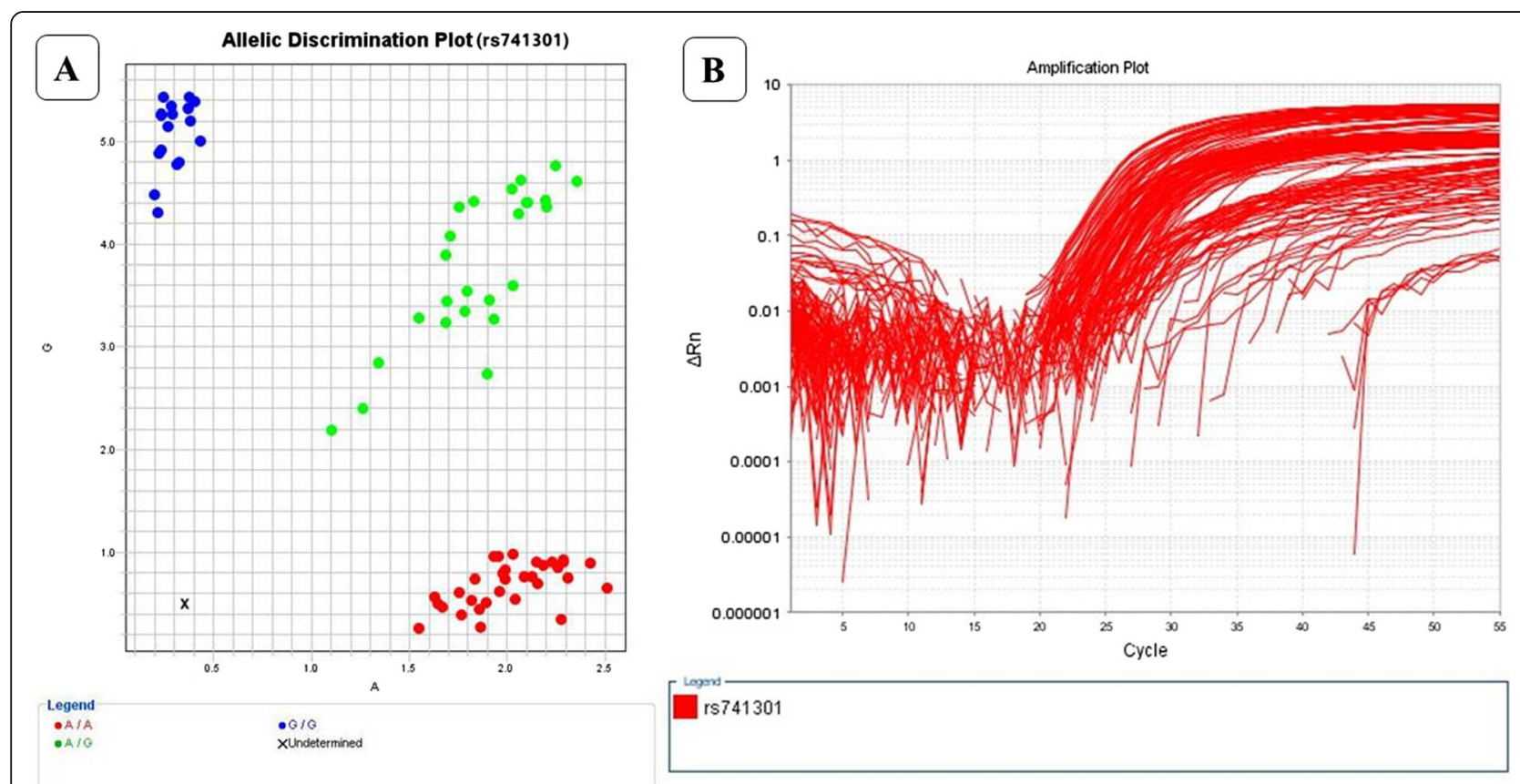

Fig. 1 a Allelic discrimination plot of ELMO1 rs741301. b Amplification plot of ELMO1 rs741301 
test was used for normally distributed data with Tukey's post hoc test, whereas the Kruskal-Wallis test was used to compare quantitative variables that were not normally distributed with Tamhane's post hoc test. Chi-square test $\left(\chi^{2}\right)$ was used to study the association between qualitative variables with the $Z$ test to compare column proportions. If any of the expected cells were less than five, Fisher's exact test was used. A two-sided $p$ value of < 0.05 was considered statistically significant. Univariate and multivariate logistic regressions models were used to identify whether BMI, WHR, waist circumference, FBS, HbA1c, cholesterol, TG, LDL, HDL, alleles, and genotypes of ELMO1 (rs741301) gene were potential independent predictors of DKD between cases and controls or between cases with DKD and cases without DKD.

\section{Results}

Clinical data of the studied groups (Table 1) showed that BMI, waist circumference, WHR, SBP, and DBP were statistically significantly high in patients $(p<0.001)$. Patients with DKD had a longer disease duration than T2DM patients without DKD $(p<0.001)$. Laboratory results (Table 2) showed that FBG, 2-hPPG, and HbA1c were significantly high in patients $(p<0.001)$, with no difference between groups 1 and 2 regarding FBG and 2hPPG $(p=0.062$ and 0.249 , resp.). Serum creatinine, ACR, eGFR, and lipid profile, except HDL-C, were significantly high in patients. AIP was significantly high in diabetic patients, with a significant difference between group 1 and group $2(p=0.008)$.

The ELMO1 rs741301 polymorphism analysis was shown in three models (Tables 3 and 4). The three

Table 1 Demographic and clinical data of the studied groups

\begin{tabular}{|c|c|c|c|c|c|}
\hline Variables & $\begin{array}{l}\text { Group } 1 \\
(n=100)\end{array}$ & $\begin{array}{l}\text { Group } 2 \\
(n=102)\end{array}$ & $\begin{array}{l}\text { Group } 3 \\
(n=102)\end{array}$ & $p$ value & Post hoc test \\
\hline \multicolumn{5}{|l|}{ Age (years) } & - \\
\hline Mean \pm SD & $48.78 \pm 5.00$ & $47.88 \pm 4.56$ & $47.14 \pm 6.38$ & \multirow[t]{2}{*}{0.097} & \\
\hline Median (IQR) & $48(46-51)$ & $46.5(46-51)$ & $46.5(43-54)$ & & \\
\hline \multicolumn{5}{|l|}{ Gender } & --_en \\
\hline Male & $64(64.0 \%)$ & $76(74.5 \%)$ & $66(64.7 \%)$ & \multirow[t]{2}{*}{0.201} & \\
\hline Female & $36(36.0 \%)$ & $26(25.5 \%)$ & $36(35.3 \%)$ & & \\
\hline \multicolumn{5}{|c|}{ Current smoking } & ------------- \\
\hline No & 84 (84.0\%) & $88(86.3 \%)$ & 102 (100.0\%) & \multirow[t]{2}{*}{0.015} & \\
\hline Yes & $16(16.0 \%)$ & $7(13.7 \%)$ & $0(0.0 \%)$ & & \\
\hline \multicolumn{5}{|l|}{ Duration (years) } & - \\
\hline Mean \pm SD & $7.98 \pm 1.65$ & $4.84 \pm 1.24$ & & \multirow[t]{2}{*}{$<0.001$} & \\
\hline Median (IQR) & $8(7-9)$ & $5.0(4-6)$ & ---- & & \\
\hline \multicolumn{5}{|l|}{ BMI $\left(\mathrm{kg} / \mathrm{m}^{2}\right)$} & P1 0.204 \\
\hline Mean \pm SD & $35.32 \pm 2.8$ & $34.64 \pm 2.54$ & $23.75 \pm 0.90$ & \multirow[t]{2}{*}{$<0.001$} & $\mathrm{P} 2<0.001$ \\
\hline Median (IQR) & $35.07(33.3-36.96)$ & $35.12(33.03-36.41)$ & $23.8(23.18-24.51)$ & & P3 $<0.001$ \\
\hline \multicolumn{5}{|c|}{ Waist circumference $(\mathrm{cm})$} & P1 0.058 \\
\hline Mean \pm SD & $105.62 \pm 5.70$ & $103.94 \pm 4.33$ & $75.78 \pm 2.77$ & \multirow[t]{2}{*}{$<0.001$} & $\mathrm{P} 2<0.001$ \\
\hline Median (IQR) & 107(104-109) & $104(101-106)$ & $76(74-78)$ & & P3 $<0.001$ \\
\hline \multicolumn{4}{|l|}{ Waist/hip ratio } & & P1 0.873 \\
\hline Mean \pm SD & $0.87 \pm 0.04$ & $0.88 \pm 0.04$ & $0.78 \pm 0.01$ & \multirow[t]{2}{*}{$<0.001$} & $\mathrm{P} 2<0.001$ \\
\hline Median (IQR) & $0.88(0.86-0.90)$ & $0.89(0.84-0.90)$ & $0.78(0.77-0.79)$ & & P3 $<0.001$ \\
\hline \multicolumn{4}{|l|}{ SBP $(\mathrm{mmHg})$} & & $\mathrm{P} 1<0.001$ \\
\hline Mean \pm SD & $129.96 \pm 7.16$ & $121.72 \pm 6.16$ & $111.96 \pm 8.62$ & \multirow[t]{2}{*}{$<0.001$} & $\mathrm{P} 2<0.001$ \\
\hline Median (IQR) & $130(130-135)$ & $120(120-125)$ & $110(100-120)$ & & P3 $<0.001$ \\
\hline \multicolumn{4}{|l|}{$\mathrm{DBP}(\mathrm{mmHg})$} & & P1 $<0.001$ \\
\hline Mean \pm SD & $85.74 \pm 5.97$ & $80.00 \pm 4.89$ & $74.50 \pm 5.31$ & \multirow[t]{2}{*}{$<0.001$} & P2 $<0.001$ \\
\hline Median (IQR) & 85 (80-90) & $80(80.0-82)$ & $70(70-80)$ & & P3 $<0.001$ \\
\hline
\end{tabular}

Group 1 DKD patients, Group 2 diabetic patients without DKD, Group 3 healthy controls, $B M I$ body mass index, SBP systolic blood pressure, DBP diastolic blood pressure, $P 1$ group 1 vs group $2, P 2$ group 1 vs group $3, P 3$ group 2 vs group 3 . Statistically significant at $p \leq 0.05$ 
Table 2 Laboratory results of the studied groups

\begin{tabular}{|c|c|c|c|c|c|}
\hline Variables & $\begin{array}{l}\text { Group1 } \\
(n=100)\end{array}$ & $\begin{array}{l}\text { Group2 } \\
(n=102)\end{array}$ & $\begin{array}{l}\text { Group } 3 \\
(n=102)\end{array}$ & $P$ value of Kruskal-Wallis test & Post hoc test \\
\hline FBS (mg/dl) & & & & & P1 0.062 \\
\hline Mean \pm SD & $169.72 \pm 34.19$ & $158.62 \pm 33.76$ & $85.45 \pm 7.38$ & $<0.001$ & P2 $<0.001$ \\
\hline Median (IQR) & 177(138-188) & $156.5(128-180)$ & $87(80-90)$ & & P3 $<0.001$ \\
\hline 2-hPPG (mg/dl) & & & & & P1 0.249 \\
\hline Mean \pm SD & $273.38 \pm 52.26$ & $262.16 \pm 40.43$ & $125.49 \pm 12.32$ & $<0.001$ & P2 $<0.001$ \\
\hline Median (IQR) & $266(228-311)$ & $258(245-288)$ & 123(120-129) & & P3 $<0.001$ \\
\hline $\mathrm{HbA1c} \%$ & & & & & P1 $<0.001$ \\
\hline Mean \pm SD & $8.30 \pm 0.83$ & $7.44 \pm 0.85$ & $4.83 \pm 0.25$ & $<0.001$ & $\mathrm{P} 2<0.001$ \\
\hline Median (IQR) & $8.2(7.6-8.8)$ & $7.3(6.9-7.9)$ & $4.8(4.6-5.0)$ & & P3 $<0.001$ \\
\hline Creatinine (mg/dl) & & & & & P1 $<0.001$ \\
\hline Mean \pm SD & $1.41 \pm 0.23$ & $1.00 \pm 0.11$ & $0.86 \pm 0.11$ & $<0.001$ & $\mathrm{P} 2<0.001$ \\
\hline Median (IQR) & $1.4(1.3-1.5)$ & $1.0(0.9-1.1)$ & $0.90(0.80-1.0)$ & & P3 $<0.001$ \\
\hline eGFR $\left(\mathrm{mL} / \mathrm{min} / 1.73 \mathrm{~m}^{2}\right)$ & & & & & P1 $<0.001$ \\
\hline Mean \pm SD & $54.81 \pm 12.80$ & $84.37 \pm 10.80$ & $99.52 \pm 10.41$ & $<0.001 \dagger$ & $\mathrm{P} 2<0.001$ \\
\hline Median (IQR) & $54.65(46.55-62.84)$ & 81.78 (76.68-89.86) & $97.76(93.16-106.46)$ & & P3 $<0.001$ \\
\hline Total cholesterol (mg/dl) & & & & & P1 0.552 \\
\hline $\begin{array}{l}\text { Mean } \pm \text { SD } \\
\text { Median (IQR) }\end{array}$ & $\begin{array}{l}247.40 \pm 23.78 \\
250(225-269)\end{array}$ & $\begin{array}{l}243.72 \pm 19.80 \\
239(231-262)\end{array}$ & $\begin{array}{l}162.13 \pm 14.89 \\
161(154-176)\end{array}$ & $<0.001$ & $\begin{array}{l}\text { P2 }<0.001 \\
\text { P3 }<0.001\end{array}$ \\
\hline Triglyceride (mg/dl) & & & & & P1 0.994 \\
\hline Mean \pm SD & $181.08 \pm 20.86$ & $181.86 \pm 28.22$ & $127.84 \pm 11.20$ & $<0.001$ & $\mathrm{P} 2<0.001$ \\
\hline Median (IQR) & $183(168-196)$ & 185 (174-198) & $128(122-135)$ & & P3 $<0.001$ \\
\hline HDL-C (mg/dl) & & & & & P1 0.145 \\
\hline Mean \pm SD & $35.22 \pm 4.77$ & $36.37 \pm 3.42$ & $49.41 \pm 5.45$ & & $\mathrm{P} 2<0.001$ \\
\hline Median (IQR) & $35(32-39)$ & $38(34-39)$ & $49(45-54)$ & $<0.001$ & P3 $<0.001$ \\
\hline LDL-C (mg/dl) & & & & & P1 0.044 \\
\hline Mean \pm SD & $177.94 \pm 22.12$ & $170.25 \pm 18.47$ & $86.49 \pm 10.60$ & $<0.001$ & $\mathrm{P} 2<0.001$ \\
\hline Median (IQR) & 182(155-195.8) & $168(155-168)$ & 87 (79-98) & & P3 $<0.001$ \\
\hline AIP & & & & & P1 0.008 \\
\hline Mean \pm SD & $0.37 \pm 0.09$ & $0.34 \pm 0.05$ & $0.05 \pm 0.06$ & $<0.001$ & $\mathrm{P} 2<0.001$ \\
\hline Median (IQR) & $0.36(0.32-0.42)$ & $0.34(0.31-0.38)$ & $0.05(0.0-0.10)$ & & P3 $<0.001$ \\
\hline ACR (mg/g creatinine) & & & & & P1 $<0.001$ \\
\hline Mean \pm SD & $132.14 \pm 82.21$ & $18.09 \pm 5.72$ & $15.21 \pm 5.65$ & $<0.001$ & $\mathrm{P} 2<0.001$ \\
\hline Median (IQR) & $98(85-150)$ & $18(14-22)$ & $15(11-19)$ & & P3 0.001 \\
\hline
\end{tabular}

Group 1 DKD patients, Group 2 diabetic patients without DKD, Group 3 healthy controls, FBS fasting blood sugar, 2-hPPG 2-hour post prandial blood sugar, HbA1c hemoglobin $A 1 C$, eGFR estimated glomerular filtration rate, $L D L-C$ low-density lipoprotein cholesterol, $H D L-C$ high-density lipoprotein cholesterol, $A I P$ atherogenic index of plasma, $A C R$ albumin/creatinine ratio, $P 1$ group 1 vs group $2, P 2$ group 1 vs group $3, P 3$ group 2 vs group $3,{ }^{\dagger}$ for ANOVA test, $I Q$ interquartile range. Statistically significant at $p \leq 0.05$

models showed statistically significant differences among the three studied groups $(p=0.001, p=0.001$, and $p<$ 0.001 , resp.). The control group had significantly higher proportions of the AA genotypes than those in groups 1 and 2 in both the dominant and codominant models and had significantly higher AA+AG genotypes in the recessive model than the other two groups; meanwhile, no significant difference was observed between groups 1 and 2 in any of the gene models.
Regarding A and G allele frequencies, group 3 had a significantly higher proportion of A allele and a significantly lower proportion of $\mathrm{G}$ allele than the other two groups. However, no significant difference was observed between group 1 and group 2 regarding the $\mathrm{A}$ and $\mathrm{G}$ alleles (Table 3).

Comparing patient groups to controls, GG genotype of ELMO1 rs741301 was significantly higher $(p<0.001)$ in DKD patients and diabetic patients without DKD than in 
Table 3 ELMO1 rs741301 allele and genotype distribution in studied groups

\begin{tabular}{llll}
\hline Genotypes & $\begin{array}{l}\text { Group 1 }(\boldsymbol{n}=\mathbf{1 0 0}) \\
\text { No. (\%) }\end{array}$ & $\begin{array}{l}\text { Group 2 (n=102) } \\
\text { No. (\%) }\end{array}$ & $\begin{array}{l}\text { Group 3 (n=102) } \\
\text { No. (\%) }\end{array}$ \\
\hline $\begin{array}{llll}\text { Co-dominance model } \\
\text { AA }\end{array}$ & $38(38.0)$ & $46(45.1)$ & $64(62.7)^{*}$ \\
AG & $36(36.0)$ & $34(33.3)$ & $32(31.4)$ \\
GG & $26(26.0)$ & $22(21.6)$ & $6(5.9)$ \\
Dominant model & & & \\
AA & $38(38.0)$ & $46(45.1)$ & $64(62.7)^{*}$ \\
AG/GG & $62(62.0)$ & $56(54.9)$ & $38(37.3)$ \\
Recessive model & & & $96(94.1) *$ \\
AA/AG & $74(74.0)$ & $80(78.4)$ & $6(5.9)$ \\
GG & $26(26.0)$ & $22(21.6)$ & $160(78.4) *$ \\
A allele & $112(56.0)$ & $126(61.7)$ & $44(21.6) *$ \\
G Allele & $88(44.0)$ & $78(38.2)$ & $<001$ \\
\hline
\end{tabular}

Group 1 DKD patients, Group 2 diabetic patients without DKD, Group 3 healthy controls. *Significantly different than its corresponding category in the other groups. Statistically significant at $p \leq 0.05$

controls in both the codominant and recessive models. Moreover, the dominant model AG+GG was significantly higher in patients $(p=0.001)$ than in controls.

Regarding A and $\mathrm{G}$ allele frequencies, a significant difference was detected between patient groups and controls $(p<0.001)$. Patients had a significantly higher proportion of $\mathrm{G}$ allele and a significantly lower proportion of A allele than the controls (Table 4).

In the univariate logistic regression between patients and controls, the GG genotype $(\mathrm{OR}=6.095,95 \% \mathrm{CI}$ $2.456-15.125, p<0.001$ ) was found to be an independent risk factor for diabetic nephropathy. In the multivariate regression model, triglyceride was the only independent

Table 4 ELMO1 rs741301 allele and genotypes distribution in patients and controls

\begin{tabular}{|c|c|c|c|}
\hline Genotypes & $\begin{array}{l}\text { Groups } 1 \text { and } 2(n=202) \\
\text { No. }(\%)\end{array}$ & $\begin{array}{l}\text { Group3 }(n=102) \\
\text { No. }(\%)\end{array}$ & $P$ value \\
\hline \multicolumn{3}{|c|}{ Co-dominance model } & $<0.001$ \\
\hline AA & $84(41.6)$ & $64(62.7) *$ & \\
\hline AG & $70(34.7)$ & $32(31.4)$ & \\
\hline GG & $48(23.8) *$ & $6(5.9)$ & \\
\hline \multicolumn{3}{|c|}{ Dominant model } & 0.001 \\
\hline AA & $84(41.6)$ & $64(62.7)$ & \\
\hline AG/GG & $118(58.4)$ & $38(37.3)$ & \\
\hline \multicolumn{3}{|c|}{ Recessive model } & $<0.001$ \\
\hline AA/AG & $154(76.2)$ & $96(94.1)$ & \\
\hline GG & $48(23.8)$ & $6(5.9)$ & \\
\hline A allele & $238(58.9)$ & $160(78.4)$ & $<0.001$ \\
\hline G Allele & $166(41.1)$ & $44(21.6)$ & \\
\hline
\end{tabular}

Groups 1 and 2 DKD patients and diabetic patients without DKD, Group 3 healthy controls. *Significantly different than its corresponding category in the other groups Statistically significant at $p \leq 0.05$ risk factor significantly associated with diabetic nephropathy (Table 5).

In the univariate logistic regression between DKD and non-DKD, HbA1c (OR $=3.252,95 \%$ CI 2.18-4.837, $p<$ $0.001)$ and HDL-C $(\mathrm{OR}=0.850,95 \%$ CI $0.788-0.916$, $p<0.001)$ were considered independent factors in both groups (Table 6).

\section{Discussion}

DKD is a severe common complication of DM. Almost $20-40 \%$ of patients with T2DM will develop DKD, and many will progress further to ESRD [25]. The pathogenesis of DKD is not fully understood until now. During the last few years, the genetic basis of DKD has been proven by GWAS and other replication studies [26]. Upregulation of ELMO1 gene expression is associated with a high blood glucose level. A mechanism that may enhance the production of ECM genes and downregulation of metalloproteinase gene and cell adhesion, which may lead to the progression of T2DM to DKD, was found. ELMO1 was suggested to initiate RAC1 integration with Dock180, leading to the upregulation of the ECM genes [27].

In this research, we investigated the rs741301 polymorphism in intron 18 of ELMO1 as a candidate gene for susceptibility to DKD in Egyptian T2DM patients.

The anthropometric measures were statistically significantly high in diabetic patients. Chandra et al. [28] also, observed no significant difference in BMI and WHR between DKD and non-DKD. Disease duration was significantly longer in group 1 than group 2, in agreement with the findings of $\mathrm{Wu}$ et al. and Bayoumy et al. [16, 29], who found a significant relationship between disease duration and DKD. Long duration of diabetes had a 
Table 5 Univariate and multivariate logistic regression among patients and controls

\begin{tabular}{|c|c|c|c|c|c|c|c|c|c|c|}
\hline \multirow[t]{3}{*}{ Variables } & \multicolumn{5}{|c|}{ Univariate } & \multicolumn{5}{|c|}{ Multivariate } \\
\hline & \multirow[t]{2}{*}{$\overline{O R}$} & \multirow{2}{*}{$\begin{array}{l}P \\
\text { value }\end{array}$} & \multirow[t]{2}{*}{ OR } & \multicolumn{2}{|l|}{$95 \% \mathrm{Cl}$} & \multirow[t]{2}{*}{$\overline{O R}$} & \multirow{2}{*}{$\begin{array}{l}P \\
\text { value }\end{array}$} & \multirow[t]{2}{*}{ OR } & \multicolumn{2}{|l|}{$95 \% \mathrm{Cl}$} \\
\hline & & & & Lower & Upper & & & & Lower & Upper \\
\hline BMI $\left(\mathrm{kg} / \mathrm{m}^{2}\right)$ & 8.836 & 0.990 & --- & --- & --- & ---- & ---- & $-\bar{y}^{---}$ & ----- & ----- \\
\hline Waist/hip ratio & 1573.291 & 0.982 & --- & --- & --- & ---- & & ---- & ---- & ---- \\
\hline Waist circumference $(\mathrm{cm})$ & 2.449 & 0.992 & --- & --- & --- & ---- & ----- & ---- & ---- & ----- \\
\hline FBS (mg/dl) & 2.909 & 0.979 & --- & --- & --- & ---- & ---- & ---- & ---- & ---- \\
\hline HbA1c \% & 42.688 & 0.986 & --- & ---- & --- & ----- & ---- & ---- & ---- & ----- \\
\hline Cholesterol (mg/dl) & 1.373 & 0.989 & --- & --- & --- & ---- & ---- & ---- & ----- & ---- \\
\hline Triglyceride(mg/dl) & 0.122 & $<0.001$ & 1.130 & 1.087 & 1.175 & 0.118 & $<0.001$ & 1.126 & 1.095 & 1.157 \\
\hline LDL-C (mg/dl) & 0.859 & 0.992 & --- & --- & --- & ---- & ----- & ----- & ----- & ----- \\
\hline HDL-C (mg/dl) & -16.401 & 0.980 & --- & ---- & --- & ----- & ----- & ---- & ----- & ----- \\
\hline Genotype* & & $<0.001$ & & & & & 0.065 & & & \\
\hline AG & 0.511 & 0.059 & 1.667 & 0.981 & 2.831 & 0.159 & 0.758 & 1.172 & 0.427 & 3.21 \\
\hline GG & 1.808 & $<0.001$ & 6.095 & 2.456 & 15.125 & 1.855 & 0.021 & 6.394 & 1.326 & 30.83 \\
\hline A allele & -1.607 & $<0.001$ & 0.201 & 0.083 & 4.86 & ---- & ---- & ---- & ---- & ----- \\
\hline G allele & 0.861 & 0.001 & 2.366 & 1.450 & 3.859 & ---- & ---- & ----- & ---- & ---- \\
\hline
\end{tabular}

$B M I$ body mass index, FBS fasting blood sugar, $H b A 1 c$ hemoglobin $A 1 C, L D L-C$ low-density lipoprotein cholesterol, $H D L-C$ high-density lipoprotein cholesterol, OR odds ratio, $\mathrm{Cl}$ confidence interval. *AA genotype was the reference. The univariate analysis was performed after that the genotype was adjusted for the TG

significant effect on kidney functions and electrolytes levels and is considered a risk factor for DKD. The relationship between DKD and duration of diabetes is explained by the fact that prolonged exposure to hyperglycemia causes damage to the glomerulus, tubule-

Table 6 Univariate logistic regression among DKD and diabetes without DKD

\begin{tabular}{|c|c|c|c|c|c|}
\hline \multirow[t]{3}{*}{ Variables } & \multicolumn{5}{|c|}{ Univariate } \\
\hline & \multirow[t]{2}{*}{ OR } & \multirow{2}{*}{$\begin{array}{l}P \\
\text { value }\end{array}$} & \multirow[t]{2}{*}{ OR } & \multicolumn{2}{|l|}{$95 \% \mathrm{Cl}$} \\
\hline & & & & Lower & Upper \\
\hline BMI $\left(\mathrm{kg} / \mathrm{m}^{2}\right)$ & 0.095 & 0.074 & 1.100 & 0.991 & 1.222 \\
\hline Waist/hip ratio & 7.163 & 0.085 & -- & --- & --- \\
\hline Waist circumference $(\mathrm{cm})$ & 0.174 & 0.001 & 1.190 & 1.105 & 1.282 \\
\hline FBS (mg/dl) & 0.010 & 0.024 & 1.010 & 1.001 & 1.019 \\
\hline $\mathrm{HbA} 1 \mathrm{c} \%$ & 1.179 & $<0.001$ & 3.252 & 2.186 & 4.837 \\
\hline Cholesterol (mg/dl) & 0.008 & 0.233 & 1.008 & 0.995 & 1.021 \\
\hline Triglyceride (mg/dl) & -0.001 & 0.822 & 0.999 & 0.988 & 1.010 \\
\hline LDL-C (mg/dl) & 0.458 & 0.010 & 1.018 & 1.004 & 1.032 \\
\hline HDL-C (mg/dl) & -0.163 & $<0.001$ & 0.850 & 0.788 & 0.916 \\
\hline Genotype & & 0.568 & & & \\
\hline AG & 0.248 & 0.444 & 1.282 & 0.679 & 2.421 \\
\hline GG & 0.358 & 0.324 & 1.431 & 0.702 & 2.916 \\
\hline A allele & 0.245 & 0.460 & 1.278 & 0.667 & 2.447 \\
\hline G allele & 0.293 & 0.307 & 1.340 & 0.764 & 2.350 \\
\hline
\end{tabular}

$B M I$ body mass index, FBS fasting blood sugar, $H b A 1 c$ hemoglobin $A 1 C, L D L-C$ low-density lipoprotein cholesterol, $H D L-C$ high-density lipoprotein cholesterol, $O R$ odds ratio, $\mathrm{Cl}$ confidence interval interstitium, and vasculature either directly or through hemodynamic changes [30].

Compared to the control group, the patient groups had higher blood pressure, serum creatinine, and ACR and lower eGFR. This result is in line with that of Bayoumy et al. [29], who found a significant difference in these variables between patients and controls. Patient groups have high FBG and 2-hPPG $(p<0.001)$, while HbA1c was significantly elevated in group $1(p<0.001)$. These findings agreed with those of Mehrabzadeh et al. and Hou et al. [30, 31], who observed that DKD had higher levels of HbA1c than T2DM without DKD. $\mathrm{HbA1c}$ is a good indicator of glycemic state. Impaired glycemic control in DM plays a crucial role in rapid progression to DKD that is caused by variable hemodynamic, metabolic, or endothelial dysfunctions.

Dyslipidemia was considered a risk factor in the progression of DKD. Impaired lipoprotein lipase (LPL) activity triggers diabetic dyslipidemia; thus, during diabetes, dyslipidemia increases macrophage infiltration and excessive ECM formation in glomeruli, leading to the development of DKD [32]. Lipid profile was significantly higher in diabetic groups with no significant difference between group 1 and group 2; these results were confirmed by Wu et al. and Wang et al. [16, 33]. However, AIP was significantly high in DKD patients. Li et al. [34] observed an association between AIP and microvascular complications in T2DM patients and suggested that patients with elevated AIP are at higher risk for microalbuminuria; thus, AIP may be an early predictor of DKD. 
Different mechanisms could explain the initiation and progression of DKD by the ELMO1 gene as follows. ELMO1 increases ROS, leading to the destruction of renal tissues [35]. Another mechanism is that the genes responsible for fibrosis, such as TGF- $\beta 1$, are stimulated by ELMO1, while antifibrotic genes, such as matrix metalloproteinase genes, are inhibited. This mechanism results in deteriorating glomerulosclerosis due to an increase in the thickness of the glomerular basement membrane [35, 36]. Moreover, cyclooxygenase 2 interacts with the ELMO1 gene to initiate and sustain glomerular damage, leading to glomerulosclerosis [13]. Despite all these pathways that demonstrate the role of the ELMO1 gene in the progress of DKD, the main pathophysiology remains poorly understood.

In this study, ELMO1 rs741301 A>G for susceptibility to DKD in Egyptian individuals was screened. GG genotype and $G$ allele were significantly elevated in diabetic patients. In contrast, AA genotype and A allele were significantly higher in controls with no statistically significant differences between group 1 and group 2 regarding either genotype (GG and AG) or allele (A and G) frequency. These outcomes agreed with the results obtained by Kim et al. and Yahya et al. [17, 18], who indicated that ELMO1 rs741301 A $>\mathrm{G}$ was not the leading cause in the development of DKD in patients with T2DM. Furthermore, Yadav et al. [37] revealed that the incidence of the GG genotype and $G$ allele was higher in diabetics patients than in healthy controls. Furthermore, the difference in the GG genotype and $\mathrm{G}$ allele occurrence was not significant in the patient groups. Bodhini et al. [38] found that the frequency of the $\mathrm{G}$ allele of ELMO1 rs741301 variant was relatively higher in the DM patients than in DKD patients, indicating that the A allele is the nonrisk allele and the G allele is the risk allele. These findings were not in line with those of Bayoumy et al. [29], who observed that ELMO1 rs741301 $\mathrm{A}>\mathrm{G}$ was a candidate variant in genetic predisposition to DKD. GG genotype was substantially correlated with $\mathrm{DKD}(\mathrm{OR}=2.7,95 \% \mathrm{CI} 1.4-5.3, p=0.016)$. The high-risk allele $\mathrm{G}$ had the following values: $\mathrm{OR}=1.9,95 \%$ CI 1.5-2.9, $p<0.001$, suggesting that ELMO1 could be a valuable target for new drugs to aid in the prevention and treatment of DKD. Hou et al. and Mohammed et al. [31, 39] confirmed that homozygous mutant GG genotype and $G$ allele of rs741301 were significant risk predictors of predisposition to DKD in T2DM patients.

Mehrabzadeh et al. [30] clarified that the $G$ allele of the ELMO1 variant rs741301 was strongly associated with DKD in patients compared to healthy controls (G allele: $\mathrm{OR}=1.7,95 \%$ CI $1.17-2.63, p=0.005$; GG genotypes: $\mathrm{OR}=2.5,95 \% \mathrm{CI} 1.2-5.4, p=0.01$ ).

However, Wu et al. [16] indicated that genetic associations at ELMO1 were observed in some independent and ethnically different groups of patients with DKD as they discovered that the A allele, not the G allele, was associated with a strong predisposing risk factor for DKD in the Chinese population $(\mathrm{OR}=3.27,95 \% \mathrm{CI}$ $1.10-9.72, p=0.03)$.

In this study, multivariate logistic regression for progression of DKD revealed that the long duration of hyperglycemia, elevated serum creatinine, total blood cholesterol, and HbA1c carry the risk for DKD and its multifactorial etiology. This result was in agreement with that of Yadav et al. [37], who reported that serum creatinine, cholesterol, glycated hemoglobin, blood pressure, and period of diabetes were related to DKD.

\section{Conclusion}

Increase expression of the ELMO1 gene facilitates phagocytosis, with an overproduction of extracellular protein, and decreases cell adhesion, contributing to the enhancement and progression of T2DM glomerulosclerosis. However, our results could not establish an association between ELMO1 rs741301 polymorphism and the development of DKD in Egyptian patients with T2DM.

The conflicts between the results could be due to the complexity of DKD pathogenesis, genetic factors, environmental factors, and small sample size. Further research, including larger sample size and more ELMO1 gene SNPs, is warranted to achieve more conclusive results about the distribution of the ELMO1 gene in Egyptian patients with T2DM.

\section{Abbreviations \\ 2-hPPG: 2-hour post prandial blood sugar; ACR: Albumin creatinine ratio; AIP: Atherogenic index of plasma; DKD: Diabetic kidney disease; ECM: Extracellular matrix; eGFR: Estimated glomerular filtration rate; ELMO1: Engulfment and cell motility 1; ESRD: End-stage renal disease; FBS: Fasting blood sugar; HbA1c: Hemoglobin A1C; HDL-C: High-density lipoprotein cholesterol; LDL-C: Low-density lipoprotein cholesterol; SNP: Single nucleotide polymorphism; T2DM: Type2 diabetes mellitus}

\section{Acknowledgements}

None

\section{Authors' contributions}

TO: Selected the idea of the study and designed the protocol of the work, and wrote the Abstract and Discussion sections of the manuscript. SZ: Collected the samples from the Endocrine Unit of Internal Medicine Department and helped in writing the Introduction section of the manuscript. MG: Edited the manuscript. KR: Wrote the practical part of the study (real-time PCR). DE: Wrote the Statistical analysis section of the Results' section and wrote the Methodology section of the manuscript.The authors have read and approved the final manuscript.

\section{Funding}

This study was self-funded from the authors and supported by the Faculty of Medicine Menoufia University facilities.

Availability of data and materials

The data that support the findings of this study are available upon request from the corresponding author. 


\section{Declarations}

\section{Ethics approval and consent to participate}

This research was approved by the Research Ethics Committee at Menoufia Faculty of Medicine according to the 1964 Helsinki Declaration, and informed written consent was taken from every participant in the study. The committee's reference number is not available for now.

\section{Consent for publication}

Consent to publish from the patient has been taken.

\section{Competing interests}

The authors declare that they have no competing interests.

\section{Author details}

'Clinical Pathology Department, Faculty of Medicine, Menoufia University, Shebin El-Kom, Egypt. ${ }^{2}$ Internal Medicine Department, Faculty of Medicine, Menoufia University, Shebin El-Kom, Egypt. ${ }^{3}$ Faculty of Medicine, Menoufia University, Shebin El-Kom, Egypt.

\section{Received: 29 December 2020 Accepted: 8 April 2021}

\section{Published online: 22 May 2021}

\section{References}

1. International Diabetes Federation "IFD", 2019. IDF Diabetes Atlas, 9th edition. Brussels, Belgium. http://www.diabetesatles.org.

2. Wei L, Xiao Y, Li L, Xiong X, Han Y, Zhu X, Sun L The susceptibility genes in diabetic nephropathy. Kidney Dis 2018; 4:226-237. https://doi.org/10.1159/ 000492633,4

3. Vallon V, Komers R. Pathophysiology of the diabetic kidney. Compr Physiol 2011;1(3):1175-1232. https://doi.org/10.1002/cphy.c100049.

4. Kanwar YS, Wada J, Sun L, Xie P, Wallner El, Chen S, Chugh S, Danesh FR Diabetic nephropathy: mechanisms of renal disease progression. Exp Biol Med (Maywood) 2008;233(1):4-11. https://doi.org/10.3181/0705-MR-134.

5. Jha JC, Banal C, Chow BS, Cooper ME, Jandeleit-Dahm K. Diabetes and kidney disease: role of oxidative stress. Antioxid Redox Signal. 2016;25(12): 657-684. https://doi:https://doi.org/10.1089/ars.2016.6664.

6. Donate-Correa J, Luis-Rodríguez D, Martín-Núñez E, Tagua V G, Hernández Carballo C, Ferri C, Rodríguez-Rodríguez A E, Mora-Fernández C, NavarroGonzález J F Inflammatory targets in diabetic nephropathy. J Clin Med 2020; 9(2):458. https://doi.org/10.3390/jcm9020458.

7. Mahwish U M, Ponnaluri K C, Heera B, Alavala S R, Devi KR, Raju S B, et al. Link between ACE I/D gene polymorphism and dyslipidemia in diabetic nephropathy: a case-control study from hyderabad. Indian J Nephrol 2020 30(2):77-84. https://doi.org/10.4103/ijn.IJN_244_18

8. Gu HF. Genetic and epigenetic studies in diabetic kidney disease. Front Genet 2019; 10: 507. https://doi.org/10.3389/fgene.2019.00507.

9. Ma J, Pei Y, Xue P, Wang Y, Bao X, Li Y. Association of the Polymorphisms in FOXO1 gene and diabetic nephropathy risk. Artif Cells Nanomed Biotechnol 2019; 47(1):1471-1475. https://doi.org/10.1080/21691401.2019.1601103.

10. Hanson RL, Millis MP, Young NJ, Kobes S, Nelson RG, Knowler WC, DiStefano JK ELMO1 variants and susceptibility to diabetic nephropathy in American Indians. Mol Genet Metab 2010;101(4):383-390. https://doi.org/10.1016/j. ymgme.2010.08.014

11. Sharma KR, Heckler K, Stoll S J, Hillebrands J, Kynast K, Herpel E, et al. ELMO1 protects renal structure and ultrafiltration in kidney development and under diabetic conditions. Sci Rep 2016; 6:37172. https://doi.org/10.103 8/srep37172, 1.

12. Goli F, Karimi J, Khodadadi I, Tayebinia H, Kheiripour N, Hashemnia M, Rahimi R. Silymarin attenuates ELMO-1 and KIM-1 expression and oxidative stress in the kidney of rats with type 2 diabetes. Indian J Clin Biochem. 2019;34(2):172-179. https://doi.org/10.1007/s12291-018-0735-0.

13. Shimazaki A, Kawamura Y, Kanazawa A, Sekine A, Saito S, Tsunoda T, Koya D, Babazono T, Tanaka Y, Matsuda M, Kawai K, liizumi T, Imanishi M, Shinosaki T, Yanagimoto T, Ikeda M, Omachi S, Kashiwagi A, Kaku K, Iwamoto Y, Kawamori R, Kikkawa R, Nakajima M, Nakamura Y, Maeda S Genetic variations in the gene encoding ELMO1 are associated with susceptibility to diabetic nephropathy. Diabetes. 2005; 54:1171-1178. https:// doi.org/10.2337/diabetes.54.4.1171, 4

14. Hanson RL, Millis MP, Young NJ, Kobes S, Nelson RG, Knowler WC, DiStefano JK ELMO1 variants and susceptibility to diabetic nephropathy in American
Indians. Mol Genet Metab 2010; 101:383-390. https://doi.org/10.1016/j. ymgme.2010.08.014, 4

15. Pezzolesi MG, Katavetin P, Kure M, Poznik D, Skupien J, Mychaleckyj JC, et al. Confirmation of genetic associations at ELMO1 in the GoKinD collection supports its role as a susceptibility gene in diabetic nephropathy. Diabetes. 2009; 58:2698-2702. https://doi.org/10.2337/db09-0641.

16. Wu HY, Wang Y, Chen M, Zhang X, Wang D, Pan Y, et al. Association of ELMO1 gene polymorphisms with diabetic nephropathy in Chinese population. J Endocrinol Invest. 2013;36(5): 298-302. https://doi.org/10.3275/ 8525 .

17. Kim S, Abboud HE, Pahl MV, et al. Examination of association with candidate genes for diabetic nephropathy in a Mexican American population. Clin J Am Soc Nephrol. 2010; 5:1072-1078. https://doi.org/10.221 5/CJN.06550909.

18. Yahya MJ, Ismail PB, Nordin NB, Md Akim A B, Yusuf WSB, Adam N L B, et al. Association of CCL2, CCR5, ELMO1, and IL8 polymorphism with diabetic nephropathy in malaysian type 2 diabetic patients. Int J Chronic Dis 2019; 2019:2053015. https://doi.org/10.1155/2019/2053015, 2053013.

19. American Diabetes Association's Standards of Medical Care in Diabetes. Diabetes Care 2020; 43(1): S14-S31. https://doi.org/10.2337/dc20-S002.

20. Schneider MF, Muñoz A, Ku E, Warady BA, Furth SL, Schwartz GJ. Estimation of albumin-creatinine ratio from protein-creatinine ratio in urine of children and adolescents with CKD. Am J Kidney Dis. 2020 6: S0272-6386(20)30931-8. https://doi.org/10.1053/j.ajkd.2020.07.015.

21. KDIGO 2012 (2013) Clinical practice guideline for the evaluation and management of chronic kidney disease. Kidney Int Suppl 3(1):5-14 https:// www.kidneyinternational.org

22. Zhu X, Yu L, Zhou H, Ma Q, Zhou X, Lei T, Hu J, Xu W, Yi N, Lei S Atherogenic index of plasma is a novel and better biomarker associated with obesity: a population-based cross-sectional study in China. Lipids Health Dis 2018;17(1):37. https://doi.org/10.1186/s12944-018-0686-8.

23. Pavlov AR, Pavlova NV, Kozyavkin SA, Slesarev Al. Recent developments in the optimization of thermostable DNA polymerase for efficient application. Trends Biotechnol 2004; 22(5): 253-260. https://doi.org/10.1016/j.tibtech.2 004.02.011.

24. Bustin SA, Benes V, Garson JA, Hellemans J, Huggett J, Kubista M, Mueller R, Nolan T, Pfaffl MW, Shipley GL, Vandesompele J, Wittwer CT The MIQE guidelines: minimum information for publication of quantitative real-time PCR experiments. Clin Chem 2009;55(4): 611-622. https://doi.org/10.1373/ clinchem.2008.112797.

25. Hsieh AR, Huang YC, Yang YF, Lin HJ, Lin JM, Chang YW, et al. Lack of association of genetic variants for diabetic retinopathy in Taiwanese patients with diabetic nephropathy. BMJ Open Diabetes Res Care 2020; 8(1): e000727. https://doi.org/10.1136/bmjdrc-2019-000727.

26. Ahmad N, Jamal R, Shah SA, Gafor AHA, Murad NAA. Renin-angiotensinaldosterone system gene polymorphisms and type 2 diabetic nephropathy in asian populations: an updated meta-analysis. Curr. Diabetes Rev 2019; 15(4): 263-276. https://doi.org/10.2174/1573399814 666180709100411

27. Shimazaki A, Tanaka Y, Shinosaki T, Ikeda M, Watada H, Hirose T, Kawamori R, Maeda S ELMO1 increases expression of extracellular matrix proteins and inhibits cell adhesion to ECMs. Kidney Int 2006;70 (10): 1769-1776. https:// doi.org/10.1038/sj.ki.5001939.

28. Chandra KP, Balaji D, Anju B. Assessment of obesity and visceral fat in diabetic nephropathy patients. Int J Adv Med 2020;7(1):72-77. https://doi. org/10.18203/2349-3933.ijam20195648.

29. Bayoumy NMK, El-Shabrawi MM, Leheta OF, Abo El-Ela A EM, Omar HH. Association of ELMO1 gene polymorphism and diabetic nephropathy among egyptian patients with type 2 diabetes mellitus. Diabetes Metab Res Rev 2020; 36(5): e3299. https://doi.org/10.1002/dmrr.3299

30. Mehrabzadeh M, Pasalar P, Karimi M, Abdollahi M, Daneshpour M, Asadolahpour E, Razi F Association between ELMO1 gene polymorphisms and diabetic nephropathy in an iranian population. J Diabetes Metab Disord 2016; 15:43. https://doi.org/10.1186/s40200-016-0265-3, 1.

31. Hou Y, Gao Y, Zhang Y, Lin S, Yu Y, Yang L. Interaction between ELMO1 gene polymorphisms and environment factors on susceptibility to diabetic nephropathy in Chinese Han population. Diabetol Metab Syndr 2019; 11:97. https://doi.org/10.1186/s13098-019-0492-0, 1.

32. Kawanami D, Matoba K, Utsunomiya K. Dyslipidemia in diabetic nephropathy. Ren Replace Ther 2016; 2: 16. https://doi.org/10.1186/s41100016-0028-0, 1. 
33. Wang Y, Zhou T, Zhang O, Fei Y, Li Z, Li S, et al. Poor renal and cardiovascular outcomes in patients with biopsy-proven diabetic nephropathy. Kidney Blood Press Res 2020; 45(3):378-390. https://doi.org/1 $0.1159 / 000505919$.

34. Li Z, Huang Q, Sun L, Bao T, Dai Z. Atherogenic index in type 2 diabetes and its relationship with chronic microvascular complications. Int J Endocrinol2018;2018: ID 1765835, 9 pages. https://doi.org/10.1155/2018/1 765835

35. Hathaway CK, Chang AS, Grant R, Kim HS, Madden VJ, Bagnell CR J, et al. High Elmo1 expression aggravates and low Elmo1 expression prevents diabetic nephropathy. Proc Natl Acad Sci USA 2016; 113(8):2218-2222. https://doi.org/10.1073/pnas.1600511113

36. Pezzolesi MG, Katavetin P, Kure M, Poznik G D, Skupien J, Mychaleckyj JC, Rich SS, Warram JH, Krolewski AS Confirmation of genetic associations at ELMO1 in the GoKinD collection supports its role as a susceptibility gene in diabetic nephropathy. Diabetes 2009; 58(11): 2698-2702. https://doi.org/1 0.2337/db09-0641.

37. Yadav AK, Kumar V, Dutta P, Bhansali A, Jha V. Variations in CCR5, but not HFE, ELMO1, or SLC12A3, are associated with susceptibility to kidney disease in north Indian individuals with type 2 diabetes. J Diabetes 2014;6 (6):547-555. https://doi.org/10.1111/1753-0407.12128

38. Bodhini D, Chidambaram M, Liju S, Revathi B, Laasya D, Sathish N, Kanthimathi S, Ghosh S, Anjana R M, Mohan V, Radha V Association of rs11643718 SLC12A3 and rs741301 ELMO1 variants with diabetic nephropathy in the south Indian population. Ann Hum Genet 2016; 80(6): 336-341. https://doi.org/10.1111/ahg.12174.

39. Mohammed HJ, Al-Saegh RM, Al-Saadi NH (2020) Role of engulfment and cell motility 1 gene polymorphism in type 2 diabetic nephropathy and its association with renal biomarkers: case-control study. J Egypt Soc Nephrol Transplant 20:98-102 http://www.jesnt.eg.net/text.asp?2020/20/2/98/283242

\section{Publisher's Note}

Springer Nature remains neutral with regard to jurisdictional claims in published maps and institutional affiliations.

\section{Submit your manuscript to a SpringerOpen ${ }^{\circ}$ journal and benefit from:}

- Convenient online submission

- Rigorous peer review

- Open access: articles freely available online

- High visibility within the field

- Retaining the copyright to your article

Submit your next manuscript at $\boldsymbol{\nabla}$ springeropen.com 